\title{
Proteomic profiling analysis of postmenopausal osteoporosis and osteopenia identifies potential proteins associated with low bone mineral density
} \author{
Dingjun Hao ${ }^{\text {Corresp. } 1}$ \\ ${ }^{1}$ Department of Spine Surgery, Honghui Hospital, Xi'an Jiaotong University, Xi'an, China \\ 2 School of Electronics and Information, Northwestern Polytechnical University, Xi'an, China \\ 3 Clinical Laboratory of Honghui Hospital, Xi'an Jiaotong University, Xi'an, China \\ 4 Department of Physiology, Hunan Polytechnic of Environment and Biology, Hengyang, China \\ 5 School of Public Health, Health Science Center, Xi'an Jiaotong University, Xi'an, China \\ Corresponding Authors: Jihan Wang, Dingjun Hao \\ Email address: 513837742@qq.com, haodingjun@126.com
}

Dageng Huang ${ }^{1}$, Yangyang Wang ${ }^{2}$, Jing Lv ${ }^{3}$, Yuzhu Yan ${ }^{3}$, Ya Hu ${ }^{4}$, Cuicui Liu ${ }^{1}$, Feng Zhang ${ }^{5}$, Jihan Wang ${ }^{\text {Corresp., }}$,

Postmenopausal osteoporosis (PMOP) is a major global public health concern and older women are more susceptible to experiencing fragility fractures. Our study investigated the associations between circulating proteins with bone mineral density (BMD) in

postmenopausal women with or without low BMD (osteoporosis and osteopenia) using a tandem mass tag (TMT) labeling proteomic experiment and parallel reaction monitoring (PRM) testing. Across all plasma samples, we quantitatively measured 1,092 proteins, and the OP and normal control (NC) samples were differentiated by principal component analysis and a partial least squares-discrimination analysis model based on the protein profiling data. The differentially abundant proteins between the low BMD and NC samples mostly exhibited binding, molecular function regulator, transporter, and molecular transducer activity, and were involved in metabolic and cellular processes, stimulus response, biological regulation, immune system processes, and so forth. TMT analysis and RRM validation indicated that the expression of protein Lysozyme C (P61626) was negatively related to BMD, while the expression of proteins Glucosidase (A0A024R592) and Protein disulfideisomerase A5 (Q14554) was positively related to BMD values. Collectively, our results suggest that postmenopausal women with low BMD have a different proteomic profile or signature. Protein alterations may play an important role in the pathogenesis of PMOP, and they may act as novel biomarkers and targets of therapeutic agents for this disease. 


\section{afProteomic profiling analysis of postmenopausal osteoporosis and osteopenia identifies} potential proteins associated with low bone mineral density

Dageng Huang ${ }^{1}$, Yangyang Wang ${ }^{2}$, Jing Lv${ }^{3}$, Yuzhu Yan ${ }^{3}, \mathrm{Ya} \mathrm{Hu}^{4}$, Cuicui Liu ${ }^{1}$, Feng Zhang ${ }^{5}$, Jihan Wang ${ }^{3}$, Dingjun Hao ${ }^{1}$

${ }^{1}$ Department of Spine Surgery, Honghui Hospital, Xi'an Jiaotong University, Xi'an, China

${ }^{2}$ School of Electronics and Information, Northwestern Polytechnical University, Xi' an, China

${ }^{3}$ Clinical Laboratory of Honghui Hospital, Xi' an Jiaotong University, Xi'an, China

${ }^{4}$ Department of Physiology, Hunan Polytechnic of Environment and Biology, Hengyang, China

${ }^{5}$ School of Public Health, Health Science Center, Xi'an Jiaotong University, Xi'an, China

Corresponding authors: Jihan Wang, 513837742@qq.com; Dingjun Hao, haodingjun@126.com

\section{ABSTRACT}

Postmenopausal osteoporosis (PMOP) is a major global public health concern and older women are more susceptible to experiencing fragility fractures. Our study investigated the associations between circulating proteins with bone mineral density (BMD) in postmenopausal women with or without low BMD (osteoporosis and osteopenia) using a tandem mass tag (TMT) labeling proteomic experiment and parallel reaction monitoring (PRM) testing. Across all plasma samples, we quantitatively measured 1,092 proteins, and the OP and normal control (NC) samples were differentiated by principal component analysis and a partial least squares-discrimination analysis model based on the protein profiling data. The differentially abundant proteins between the low $\mathrm{BMD}$ and $\mathrm{NC}$ samples mostly exhibited binding, molecular function regulator, transporter, and molecular transducer activity, and were involved in metabolic and cellular processes, stimulus response, biological regulation, immune system processes, and so forth. TMT analysis and RRM validation indicated that the expression of protein Lysozyme C (P61626) was negatively related to BMD, while the expression of proteins Glucosidase (A0A024R592) and Protein disulfideisomerase A5 (Q14554) was positively related to BMD values. Collectively, our results 
suggest that postmenopausal women with low BMD have a different proteomic profile or signature. Protein alterations may play an important role in the pathogenesis of PMOP, and they may act as novel biomarkers and targets of therapeutic agents for this disease.

Keywords: Postmenopausal osteoporosis, Bone mineral density, Proteomics, TMT, PRM, Diagnostic biomarkers, Therapeutic targets

\section{INTRODUCTION}

Osteoporosis (OP) is a common metabolic bone disorder characterized by the microarchitectural deterioration of bone tissues, low bone mineral density (BMD), and increased fracture risk. Menopause and aging are two critical factors that are directly related to OP onset(Cheishvili et al., 2018). Postmenopausal osteoporosis (PMOP) is a major global public health concern that frequently presents in postmenopausal women due to estrogen deficiency and the continuous calcium loss that occurs with aging(Thaung Zaw et al., 2018). Osteoporotic fracture (OF) caused by OP is a major hazard and is one of the main causes of disability and death in older adults(Qaseem et al., 2017). It is estimated that there will be about 4.83 million OF cases in 2035 and about 5.99 million cases in 2050(Si et al., 2015). A woman's risk of developing OF during her lifetime $(40 \%)$ is greater than the risk for ovarian cancer, breast cancer, and endometrial cancer combined(Lin et al., 2015).In response to this, studies exploring novel biomarkers and the bone metabolism-related mechanisms underlying PMOP that aid in its early diagnosis are urgently needed and relevant.

At present, there is no ideal method to measure or evaluate bone strength directly. Clinically, the BMD value measured by dual-energy X-ray absorptiometry (DXA) is used to diagnose OP and predict the risk of $\mathrm{OF}($ Dimai, 2017). Bone metabolism is a dynamic process and current research has not yet clarified the pathogenesis of osteopenia $(\mathrm{ON})$ or $\mathrm{OP}$; it is generally accepted that the imbalance between osteoblast and osteoclast metabolism plays a key role in the pathogenesis of OP(Lewiecki, 2011). When bone metabolism changes, the levels of serum bone turnover markers (BTMs) can change in as early as 3 days, while BMD can change after 1 
55

56

57

month(McClung et al., 2006). Further, high-frequency detection of BMD is unrealistic, irrespective of whether the bone density value has changed. In clinical practice, the DXA-BMD measurement is usually performed every 6 months or annually for high-risk groups or for those who receive medication to monitor the natural course of the disease and evaluate the efficacy of various drugs for OP. Therefore, some biomarker levels may exhibit changes in the early stages of abnormal bone metabolism, particularly when the BMD value may not meet the criteria for osteopenia (ON) or OP, and the body fluid testing for metabolic molecules is more convenient and implementable for BMD measurement. However, there are many meaningful studies such as serum biomarkers may be associated with high bone turnover and BMD in postmenopausal women(Bhattacharyya, S. et al. 2008) and proteomic analysis reveals Vitamin D-Binding Protein (VDBP) as a potential biomarker for low BMD in Mexican postmenopausal women(MartínezAguilar MM et al. 2019). They are outstanding inspiration for our study.

When compared with the findings of a genome and transcriptome study, proteomics and metabolomics appears to be closer to the phenotype, so it is more suitable for mechanistic studies, disease typing, and biomarker discovery(Jimenez-Munguia et al., 2018; Li et al., 2019; Li \& Shui 2019; Wilmanski et al., 2019). Previous human studies have identified a few candidate metabolites for BMD alteration(Miyamoto et al., 2018; You et al., 2014), while more research about proteome studies is needed to better understand the molecular changes associated with low $\mathrm{BMD}$ in $\mathrm{OP}$ and $\mathrm{ON}$, especially in postmenopausal women who are more susceptible to experiencing fragility fractures. Herein, we performed mass spectrometry (MS)-based proteomic experiments with tandem mass tag (TMT) labeling and further validated a few candidate differentially abundant proteins (DAPs) obtained from the MS data by means of parallel reaction monitoring (PRM) analysis in postmenopausal women with normal bone mass, ON, and OP. Our aim was to identify novel candidate proteins that are associated with BMD changes in postmenopausal women with a low BMD, while also revealing the mechanism of abnormal bone metabolism and possible diagnostic biomarkers underlying this widespread disease. 
82

83

84

\section{MATERIALS \& METHODS}

\section{Study populations and design}

For this study, we recruited a total of 54 postmenopausal females, including 18 female patients with primary PMOP, 18 with postmenopausal ON, and 18 with normal bone mass as NCs were recruited from March 2018-February 2019 in Honghui Hospital, Xi'an Jiaotong University (People's Republic of China). Patients with secondary osteoporosis, including those with Cushing's syndrome, hyperparathyroidism, hyperthyroidism, steroid abuse, and other malignancies such as chronic liver disease, heart disease, and kidney disease were excluded. Drug factors including glucocorticoids, immunosuppressants, heparin, anticonvulsants, anticancer drugs, aluminum-containing antacids, thyroid hormones, etc. were also excluded. In addition, BMD measurements were not available in the following situations: (1) those orally administered with drugs within 2-6 days of measurement that affect image development; (2) when radioisotope inspections were carried out within 3 days; (3) those who cannot lie on the examination bed, or cannot adhere to the 5-min test; (4) those with a severe deformity of the spine or metal implants on the spine.Finally, biochemical detection of serum 25-hydroxyvitamin D (vitamin D), alkaline phosphatase (ALP), procollagen type $1 \mathrm{~N}$-peptide (P1NP), and Cterminal cross-linking telopeptide of type 1 collagen (CTX) concentrations as well as BMD measurements were determined for all participants. Each participant signed an informed consent form, and the project was approved by the Institutional Review Board, Honghui Hospital, Xi'an Jiaotong University (Project No: 2018-22). We collected participants' information, including their age, age at menopause, height (m), and body mass (kg). Participants' BMI was calculated as mass/height ${ }^{2}$. The experimental workflow is presented in Figure 1.

\section{BMD measurement}

DXA was used to detect the BMD of the lumbar spine $\left(\mathrm{L}_{1-4}\right)$ and hip (Discovery Wi, Hologic, Marlborough, MA, USA) in all participants. The scan images were obtained from the detector and are shown in Figure 2. We collected information pertaining to patients' BMD values $\left(\mathrm{g} / \mathrm{cm}^{2}\right)$ 
109

110

111

112

113

114

115

116

117

118

119

120

121

122

123

124

125

126

127

and $\mathrm{T}$ scores. The $\mathrm{T}$-score reference ranges were calculated using data from healthy young Asian women provided by the bone densitometry equipment manufacturer. Participants were categorized as having osteoporosis, osteopenia, or normal BMD according to the World Health Organization's T-score classification: participants with T-scores $\leq-2.5$ at any site were diagnosed as having OP, $-2.5<\mathrm{T}$-scores $<-1.0$ had $\mathrm{ON}$, and T-scores $\geq-1.0$ were indicative of normal bone mass. The parallel reaction monitoring approach has been used for BMD-related DAPs validation.

\section{Blood sample collection}

We collected the fasting venous blood samples from each participant, and the blood samples were centrifuged at $4^{\circ} \mathrm{C}$ at $3,000 \mathrm{rpm}$ for 15 minutes. After the centrifugal, the supernatant from each sample were collected and stored at $-80^{\circ} \mathrm{C}$ until measurement. In each group, we randomly mixed six plasma samples into one sample for the TMT proteome experiment and PRM validation.

\section{Biochemical detection}

Serum 25-hydroxyvitamin D (Vitamin D), alkaline phosphatase (ALP), procollagen type $1 \mathrm{~N}$ peptide (P1NP), and C-terminal cross-linking telopeptide of type 1 collagen (CTX) were detected using the Roche electrochemiluminescence system (Cobas e 601 analyzer, Roche Diagnostics GmbH, Wetzlar, Germany).

\section{Protein extraction and enzymolysis}

The plasma pools were depleted of the proteins by using the Multiple Affinity Removal LC Column-Human 14(Cat. 5188-6560, Agilent, CA, USA) according to the protocol from the manufacturer. The desalination and concentration of the low-abundance components were performed by using a $10 \mathrm{kDa}$ ultrafiltration tube. One volume of SDT (4\% SDS, $100 \mathrm{mM}$ Tris/ $\mathrm{HCl} \mathrm{pH}$ 7.6, $0.1 \mathrm{M}$ dithiothreitol) buffer was added, and then boiled for 15 minutes, then 
136

137

138

139

140

141

142

143

144

145

146

147

148

149

150

151

152

153

154

155

156

157

158

159

160

161

162

centrifuged for 20 minutes with 14,000g. The BCA Protein Assay Kit (Bio-Rad, Hercules, CA, USA) was used for protein quantification, and all the supernatant samples were stored at $-80^{\circ} \mathrm{C}$ for further measurement.

\section{SDS-PAGE Separation}

Overall, proteins $(20 \mu \mathrm{g})$ were mixed by the $5 \times$ loading buffer for each sample, respectively, and boiled for 5 minutes. Proteins from all the samples were separated by a $12.5 \%$ sodium dodecyl sulfate (SDS)-polyacrylamide gel electrophoresis (PAGE) gel and the Coomassie Blue R-250 staining system was used for binds visualization.

\section{TMT labeling}

$200 \mu \mathrm{g}$ of proteins for each sample were incorporated into $30 \mu \mathrm{l}$ SDT buffer (4\% SDS, $100 \mathrm{mM}$ DTT, $150 \mathrm{mM}$ Tris-HCl pH 8.0). The detergent, DTT and other low-molecular-weight components were removed using UA buffer (8 M Urea, $150 \mathrm{mM}$ Tris-HCl pH 8.0) by repeated ultrafiltration (Microcon units, $10 \mathrm{kD}$ ). Then $100 \mu \mathrm{l}$ iodoacetamide (100 mM IAA in UA buffer) was added to block reduced cysteine residues and the samples were incubated for 30 min in darkness. The filters were washed with $100 \mu$ UA buffer three times and then $100 \mu 100 \mathrm{mM}$ TEAB buffer twice. Finally, the protein suspensions were digested with $4 \mu \mathrm{g}$ trypsin in $40 \mu \mathrm{l}$ TEAB buffer overnight at $37^{\circ} \mathrm{C}$, and the resulting peptides were collected as a filtrate. The peptide content was estimated by UV light spectral density at $280 \mathrm{~nm}$ using an extinctions coefficient of 1.1 of $0.1 \%(\mathrm{~g} / \mathrm{l})$ solution that was calculated on the basis of the frequency of tryptophan and tyrosine in vertebrate proteins.

Following this and according to the manufacturer's instructions (Thermo Fisher Scientific, Waltham, MA, USA), a peptide mixture of each sample $(100 \mu \mathrm{g})$ was labeled withthe TMT reagent.

\section{Peptide fractionation with high $\mathrm{pH}$ reversed phase}


163

164

165

166

167

168

169

170

171

172

173

174

175

176

177

178

179

180

181

182

183

184

185

186

187

188

189

According to the manufacturer's instructions, increasing the acetonitrile step-gradient elution, the TMT-labeled digest samples into 10 fractions fractionated with a Pierce high $\mathrm{pH}$ reversed-phase fractionation kit (Thermo Fisher Scientific).

\section{Mass Spectrometry}

Each fraction was injected in order to analyze the nano-LC-MS/MS. A reverse-phase trap column which is connected to a C18reversed-phase analytical column in buffer A $(0.1 \%$ formic acid) and separated with a linear gradient of buffer B ( $84 \%$ acetonitrile and $0.1 \%$ formic acid) loaded the peptide mixture and the flow rate was $300 \mathrm{~nL} /$ minute and it was controlled by IntelliFlow technology.

The LC-MS/MS analysis was completed with a Q Exactive mass spectrometer and it was coupled to Easy nLC for 60 minutes. The mass spectrometer was performed in positive ion mode. Using a data-dependent top10 method can obtain the MS data, and the most abundant precursor ions were selected from the scan of $300-1,800 \mathrm{~m} / \mathrm{z}$. An automatic gain control (AGC) target was3e6, and the maximum inject time was $10 \mathrm{~ms}$. Dynamic exclusion duration was 40 seconds. Survey scans were obtained with the resolution of 70,000 at m/z 200 and the resolution for the HCD spectra was 17,500 with m/z 200, 35,000 at m/z 200, and the width of isolation was $2 \mathrm{~m} / \mathrm{z}$. The normalized collision energy was 30 and the under fill ratio specified the minimum percentage of the target value was defined as $0.1 \%$. The instrument was performed by using peptide recognition mode enabled. The LC-MS/MS spectra were searched by using a MASCOT engine which was embedded into Proteome Discoverer 1.4. The mass spectrometry proteomics data have been deposited to the ProteomeXchange Consortium via the PRIDE(Perez-Riverol, $Y$. et al., 2019) partner repository with the dataset identifier PXD017804.

\section{PRM analysis}

We further performed LC-PRM analysis(Peterson et al., 2012) for several selected proteins from the same samples, to test the protein abundance obtained by previous TMT analysis. We 
190

191

192

193

194

195

196

197

198

199

200

201

202

203

204

205

206

207

208

209

210

211

212

213

214

215

216

prepared the peptides and then analyzed by using TMT analysis according to the protocol, and TOMHAQ has used for validation (Shanghai Applied Protein Technology Co., Ltd., Shanghai, China)(Zhang et al., 2019). The raw data were analyzed with the bioinformatic tool Skyline(MacLean et al., 2010) (MacCoss Lab, University of Washington, Seattle, WA, USA), where the signal intensities of the peptide sequences for the significantly altered proteins were quantified relative to each sample, and normalized to the standard reference(Liu \& Lv 2019).

\section{Statistical and bioinformatics analysis}

Basic information including participants' age, age at menopause, duration of menopause, BMI, biochemical detection, BMD, and $\mathrm{T}$ score were displayed as the mean \pm standard deviation (SD). A $P$-value $<0.05$ with two-tailed Student's $t$-test was considered statistically different between two groups. A correlation analysis between basic information, protein expression, and BMD values was performed using R 3.6.0 tools and GraphPad Prism 8.0.1 statistical software (GraphPad, La Jolla, CA, USA). Proteins with a fold change (FC) $>1.2$ as well as a statistical $P$ value $<0.05$ between two groups were selected as DAPs. The unsupervised principal component analysis (PCA) and partial least squares discriminant analysis (PLS-DA) model of proteome data were used in this study. PCA and PLS-DA analysis of proteome data, and the bi-clustering and volcano plots of DAPs were performed with R 3.6.0 tools. We searched Blast2GO (version 3.3.5) during the gene ontology mapping and annotation procedure(Gotz et al., 2008). GO enrichment on three modules including biological process (GO-BP), molecular function (GO-MF), and cellular component (GO-CC) was applied based on a Fisher' exact test, with $P$-values $<0.05$ considered as statistically significant functional categories.

\section{RESULTS}

\section{Characteristics of the study population and correlation analysis}

Our study recruited a total of 54 postmenopausal females, including 18 females with normal bone mass (normal controls [NC]), 18 females with $\mathrm{ON}$, and 18 with OP. We collected and 
217 analyzed patients' information, including their age, age at menopause (M_age), body mass index 218 (BMI), biochemical detection results and BMD measurement results (BMD value and T score 219 data from the DXA-BMD detection). Figure 2 displays the DXA scan images obtained during 220 the BMD measurement using the detector. As for BMD and T scores, when comparing the ON

221

222

223

versus $\mathrm{NC}, \mathrm{OP}$ versus $\mathrm{NC}$, and $\mathrm{OP}$ versus $\mathrm{ON}$ groups, all showed statistically significant differences, which is consistent with the diagnostic criteria for OP and ON. There were no statistically significant differences between any of the two groups in terms of age, age at menopause, and serum concentration of Vitamin, ALP, P1NP and CTX, while the participants in OP group showed a significant lower BMI compared with that in NC and ON groups (Table 1).

We further performed Pearson's correlation analysis between the different indicators to explore BMD-related factors. Overall, the BMD values were negatively correlated with age (Figure 3A). Meanwhile, a negative correlation was also observed between the menopause duration and BMD values (Figure 3C), while a positive correlation was found between BMD and age at menopause (Figure 3B), and between BMD and BMI (Figure 3D). Besides, we observed a positive correlation between the concentration of Vitamin D and BMD values, although not statistically significant (Supplementary Figure 1A), and the concentration of ALP, P1NP, CTX was not significantly related with the BMD values (Supplementary Figures 1B-1D).

\section{Differentially abundant protein (DAP) analysis in OP, ON, and among normal controls} with proteomic data

Before the proteomics experiment, we randomly mixed six samples into one sample in each group; that is, three mixed plasma samples in each group were prepared for further proteome analysis. High-throughput, label-free, liquid chromatography-tandem mass spectrometry (LCMS/MS) experiments identified a total of 1,092 proteins in the plasma samples in our study. The unsupervised principal component analysis (PCA) and partial least squares discriminant analysis (PLS-DA) model of proteome data revealed evidence of the separation between low BMD (OP and $\mathrm{ON}$ ) and the $\mathrm{NC}$, especially when discriminating between $\mathrm{OP}$ and $\mathrm{NC}$ samples, while it 
244 showed less-than-ideal effects on the separation between the OP and ON samples (Figures 4A245 4B). We selected the proteins with a fold change (FC) $>1.2$ (i.e., where upregulation was $>1.2$ 246 times or downregulation $<0.83$ times), and $P<0.05$ indicated a statistically significant difference 247 in the DAPs between two compared groups. When compared with the NC group, there were 17 248 upregulated and 20 downregulated DAPs in the ON group (Figures 4C-4D), and 32 upregulated 249 and 123 downregulated DAPs in the OP group (Figures 4C and 4E). Compared with the ON 250 group, there were 11 upregulated and 5 downregulated DAPs in the OP group (Figures 4C and 251 4F). If we set the $\mathrm{FC}>1.5$ (i.e., when the upregulation was $>1.5$ times or downregulation $<0.67$ 252 times), $P<0.5$ as the criteria for DAPs, there were 8,127 , and 3 DAPs in the ON versus NC, OP versus NC, and $\mathrm{OP}$ versus $\mathrm{ON}$ groups, respectively (Figure 4C). The details of the protein quantification results and differential analysis between each group comparison are presented in Supplementary Files 1 (ON versus NC), Supplementary Files 2 (OP versus NC) and

256 257

Supplementary Files 3 (OP versus $\mathrm{ON}$ ). The peptide lists of all the identified proteins in the study are presented in Supplementary Files 4.

These results may suggest that the proteome of OP samples were different for the NC group, whereas the ON group (the middle group) showed greater similarity with the OP group, as there was a partly overlapping PLS-DA model between the OP and ON groups and the number of DAPs was less in the OP versus ON group when compared with the ON versus NC group. Thus, in subsequent analyses, we placed greater focus on the difference between low BMD (OP and $\mathrm{ON}$ ) and NC. Functional analysis indicated that the DAPs in the ON versus NC groups mainly demonstrated catalytic, binding, and molecular function regulator activity; the DAPs appeared to be involved in metabolic, cellular, stimulus response, biological regulation, and immune system processes (Figure 5A). Similarly, the DAPs in the OP versus NC groups mostly exhibited binding, molecular function regulator, transporter, and molecular transducer activity, and appeared to be involved in cellular, stimulus response, biological regulation, metabolic, and biological regulation processes (Figure 5B). 
271

272

273

274

275

276

277

278

279

280

281

282

283

284

285

286

287

288

289

290

291

292

293

294

295

296

297

\section{Identification of candidate proteins correlated with low BMD values in PMOP}

In our study, most of the DAPs existed in the OP versus NC groups, followed by the ON versus $\mathrm{NC}$ and OP versus ON groups. We focused on the DAPs in the low BMD group, which was found in both the $\mathrm{OP}$ versus $\mathrm{NC}$ and $\mathrm{ON}$ versus $\mathrm{NC}$ comparisons. When compared with the normal BMD samples, seven proteins were differentially expressed in the OP and ON groups, including Adipocyte plasma membrane-associated protein (H0Y512), Protein disulfideisomerase A5 (Q14554), cDNA FLJ51711 (B4DE30), Glucosidase (A0A024R592), Lysozyme C (P61626), Uncharacterized protein DKFZp666N164 (Q658S4) and Complement C4-A (P0C0L4) (Table 2). Supplementary Figure 2 showed the MS/MS spectra for the seven DAPs in the low BMD groups. Bi-clustering analysis of these seven DAPs indicated a clear discrimination between normal BMD and low BMD samples (Figure 6A). The detailed expression of the seven proteins in each group were showed in Figure 6B-6H. The expression of protein protein Lysozyme C was positively related with BMD values (Supplementary Figure 3A), while the expression of proteins proteins Glucosidase, cDNA FLJ51711, and Protein disulfideisomerase A5 was negatively related with BMD values (Supplementary Figures 3B-3D). The correlation between the expression of Complement C4-A, Uncharacterized protein DKFZp666N164, Adipocyte plasma membrane-associated protein, and BMD values was not highly significant (Supplementary Figures 3E-3G). The reported numbers of proteins were supported with more than 1 peptide.

\section{Verification of the candidate protein biomarkers associated with low BMD with PRM}

In this study, Adipocyte plasma membrane-associated protein, Protein disulfideisomerase A5, cDNA FLJ51711, Glucosidase, Lysozyme C, Uncharacterized protein DKFZp666N164, and Complement C4-Awere differentially expressed in OP and ON group compared with normal sample. However the correlation between the expression of Complement C4-A, Uncharacterized protein DKFZp666N164, Adipocyte plasma membrane-associated protein, and BMD values was not highly significant. Therefore we further selected the four BMD-related DAPs including Lysozyme C, Glucosidase, cDNA FLJ51711, and Protein disulfideisomerase A5 for validation 
298

299

300

301

302

303

304

305

306

307

308

309

310

311

312

313

314

315

316

317

318

319

320

321

322

323

324

using the parallel reaction monitoring approach. Among them, the abundance of Lysozyme C was also decreased in the low BMD groups, while Glucosidase and Protein disulfideisomerase A5 expression were increased as BMD decreased (Figure 7). These results from the PRM testing suggested that the candidate proteins demonstrated similar trends as the TMT results, which confirmed the credibility of the proteomics data and verified the DAPs between the NC and low BMD samples.

\section{DISCUSSION}

The risk of developing OP and broken bones is increasing in association with advanced age. Several factors have contributed to the result that women are more likely to develop OP than men. First, women tend to have smaller, thinner bones than men(Cooper et al., 2007). Second, estrogen levels decline dramatically when women reach menopause, which results in consequent bone loss(Ikeda et al., 2019). This explains why the chance of developing OP increases once a woman reaches menopause and why that risk increases with age.

During the process of bone remodeling, biochemical products are produced and can be detected in the blood and/or urine, which are considered to be biomarkers. Plasma and serum are rich sources of information regarding an individual's health state, and protein tests inform medical decision making(Geyer et al., 2019; Lin et al., 2018). Biomedical substances that are involved in metabolic activities are tied to an individual's biological or metabolic status; thus, circulating proteins are considered sensitive and specific markers of certain pathological states, including abnormal bone metabolism(Brosseron et al., 2018; Shao et al., 2019). Thus far, no studies have been reported that directly focused on the plasma proteome in Chinese PMOP females. There are several studies that have analyzed the quantitative proteome profiles and explored particular BMD-related proteins or genes based on peripheral blood monocytes(Shao et al., 2019; Zhang et al., 2016). Recently, a comparative proteomics analysis of serum microvesicles for the evaluation of OP was reported, and the authors found that Profilin 1 could differentiate those in the OP group from those in the ON and normal groups(Huo et al., 2019). 
Our study is the first to investigate plasma proteomic profiling in Chinese postmenopausal women with OP, ON, and normal bone mass using TMT and PRM approaches. Overall, by means of the PCA and PLS-DA model, a relative separation between the low BMD and NC groups was found, and the proteome expression patterns in the ON samples demonstrated greater similarity with the OP samples, as compared with the NC samples, which indicates that the alteration of an individual's plasma proteome profile is sensitive and specific to BMD changes, thus making it possible to discriminate between postmenopausal women with low BMD from those with normal bone mass. The number of DAPs between each of the two groups also confirmed the above conclusion, that OP versus NC had the most numerous DAPs, followed by $\mathrm{ON}$ versus $\mathrm{NC}$ and $\mathrm{OP}$ versus $\mathrm{ON}$ (Figures $4 \mathrm{C}-4 \mathrm{~F}$ ). Combing the TMT proteome data and PRM validation testing, we finally identified three candidate proteins that correlated with BMD values (Figures 6 and 7). This provides the basis for identifying novel biomarkers and exploring the pathogenesis related to abnormal bone metabolism in the field of PMOP research. In addition, there were no statistically significant differences between any of the two groups in terms of age and age at menopause, while the participants in OP group showed a significant lower BMI compared with that in NC and ON groups (Table 1). Therefore we conclude that the proteins observed were not due to differences in the BMI between the groups studied.

The specific effects of protein lysozyme C, glucosidase and protein disulfideisomerase A5 on osteoporosis are still unclear. Studies have reported the correlation with low BMD/osteoporosis and the late-onset Pompe disease (LOPD)(Papadimas et al., 2011; Sheng et al., 2017). LOPD is a lysosomal storage disease resulted from deficiency of the enzyme acid $\alpha$ glucosidase, and enzyme replacement therapy (ERT) with alglucosidase alfa is the only specific treatment available(van der Ploeg et al., 2010). In a recent research, patients with LOPD showed improvement in BMD after alglucosidase ERT(Sheng et al., 2017). Protein disulfideisomerase A5 belongs to the protein disulfideisomerase (PDI) family. Studies demonstrated that some murine models for osteoporosis is related to endoplasmic reticulum (ER) stress response of osteoblasts(Hino et al., 2010; Li et al., 2018). The ER molecular chaperones including PDI are 
352 down-regulated in osteoblasts from osteoporosis patients(Hino et al., 2010). Icariin (ICA, a 353 flavonol glycoside isolated from a traditional Chinese medicinal herb Epimedium sagittatum) 354 have demonstrated its anti-osteoporotic and osteogenic differentiation effects(Chen et al., 2005; 355 Meng et al., 2005). Recently, secretome analysis demonstrated an upregulation of the expression 356 of protein disulfideisomerase family A, member 3 (PDIA3) after ICA treatmentinduced 357 osteogenesis in rat osteoblasts(Qian et al., 2018). Considering the above reports and the results 358 from our study together, glucosidase and protein disulfideisomerase may be potential targets of therapeutic agents for the prevention of bone loss in the low BMD patients.

In this study, we identified differentially expressed proteins in OP and ON samples when compared to normal controls. Additionally, a selected subgroup of differentially expressed proteins was further validated using the PRM approach. However, it's restricted to the number of clinical sample and follows up study, what we identified in this study are very primary. And further validation for our findings need to be completed both in lab and in clinic. And the specificity and sensitivity of candidate biomarkers we found also need to be improved.

\section{CONCLUSIONS}

In conclusion, the study presented a proteome profiling analysis using TMT and PRM approaches obtained from postmenopausal females with OP, ON, and normal bone mass. The PLS-DA model of the protein expression data clearly distinguished OP from the normal samples. We also identified the differentiating abundant proteins, some of which showed significant correlations with BMD values. These data suggest that these protein profiles are indicators of bone metabolism status; the candidate proteins are associated with decreasing BMD values and may presumably represent an increased risk of fracture. These markers may provide information that guides the monitoring of both disease progression and treatment efficacy for PMOP females.

\section{ACKNOWLEDGMENTS}

377 The authors are grateful to the Shanghai Applied Protein Technology (Shanghai, China) for 378 providing technical assistance with data analysis. 


\section{REFERENCES}

Bhattacharyya, S.; Siegel, E.R.; Achenbach, S.J.; Khosla, S.; Suva, L.J. 2008.Serum biomarker profile associated with high bone turnover and BMD in postmenopausal women. J Bone Miner Res 23:1106-1117DOI: 10.1359/jbmr.080235.

Brosseron F, Traschutz A, Widmann CN, Kummer MP, Tacik P, Santarelli F, Jessen F, and Heneka MT. 2018. Characterization and clinical use of inflammatory cerebrospinal fluid protein markers in Alzheimer's disease. Alzheimers Res Ther 10:25DOI: 10.1186/s13195-018-0353-3.

Cheishvili D, Parashar S, Mahmood N, Arakelian A, Kremer R, Goltzman D, Szyf M, and Rabbani SA. 2018. Identification of an Epigenetic Signature of Osteoporosis in Blood DNA of Postmenopausal Women. $J$ Bone Miner Res 33:1980-1989DOI: 10.1002/jbmr.3527.

Chen KM, Ge BF, Ma HP, Liu XY, Bai MH, and Wang Y. 2005. Icariin, a flavonoid from the herb Epimedium enhances the osteogenic differentiation of rat primary bone marrow stromal cells. Pharmazie 60:939-942.

Cooper DM, Thomas CD, Clement JG, Turinsky AL, Sensen CW, and Hallgrimsson B. 2007. Age-dependent change in the 3D structure of cortical porosity at the human femoral midshaft. Bone 40:957-965.

Dimai HP. 2017.Use of dual-energy X-ray absorptiometry (DXA) for diagnosis and fracture risk assessment; WHOcriteria, T- and Z-score, and reference databases. Bone 104:39-43DOI: 10.1016/j.bone.2016.

Geyer PE, Voytik E, Treit PV, Doll S, Kleinhempel A, Niu L, Muller JB, Buchholtz ML, Bader JM, Teupser D, Holdt LM, and Mann M. 2019. Plasma Proteome Profiling to detect and avoid sample-related biases in biomarker studies.EMBO Mol Med 11:e10427DOI: 10.15252/emmm.201910427.

Gotz S, Garcia-Gomez JM, Terol J, Williams TD, Nagaraj SH, Nueda MJ, Robles M, Talon M, Dopazo J, and Conesa A. 2008. High-throughput functional annotation and data mining with the Blast2GO suite. Nucleic Acids Res 36:3420-3435DOI: 10.1093/nar/gkn176.

Hino S, Kondo S, Yoshinaga K, Saito A, Murakami T, Kanemoto S, Sekiya H, Chihara K, Aikawa Y, Hara H, Kudo T, Sekimoto T, Funamoto T, Chosa E, and Imaizumi K. 2010.Regulation of ER molecular chaperone prevents bone loss in a murine model for osteoporosis. J Bone Miner Metab 28:131-138DOI: 10.1007/s00774-0090117-z.

Huo C, Li Y, and Qiao Z. 2019.Comparative proteomics analysis of microvesicles in human serum for the evaluation of osteoporosis.Electrophoresis 40:1839-1847DOI: 10.1002/elps.201900130.

Ikeda K, Horie-Inoue K, and Inoue S. 2019.Functions of estrogen and estrogen receptor signaling on skeletal muscle. J Steroid Biochem Mol Biol 191:105375DOI: 10.1016/j.jsbmb.2019.105375.

Jimenez-Munguia I, Calderon-Santiago M, Rodriguez-Franco A, Priego-Capote F, and Rodriguez-Ortega MJ. 2018. Multi-omic profiling to assess the effect of iron starvation in Streptococcus pneumoniae TIGR4. PeerJ 6:e4966DOI: 10.7717/peerj.4966.

Lewiecki EM. 2011.New targets for intervention in the treatment of postmenopausal osteoporosis. Nat Rev Rheumato/7:631-638DOI: 10.1038/nrrheum.2011.130.

Li FH, Sun L, Wu DS, Gao HE, and Min Z. 2019.Proteomics-based identification of different training adaptations of aged skeletal muscle following long-term high-intensity interval and moderate-intensity continuous training in aged rats. Aging (Albany NY) 11:4159-4182DOI: 10.18632/aging.102044.

Li H, Li D, Ma Z, Qian Z, Kang X, Jin X, Li F, Wang X, Chen Q, Sun H, and Wu S. 2018. Defective autophagy in 
osteoblasts induces endoplasmic reticulum stress and causes remarkable bone loss. Autophagy 14:17261741DOI: 10.1080/15548627.2018.1483807.

Li S, and Shui W. 2019.Systematic mapping of protein-metabolite interactions with mass spectrometry-based techniques. Curr Opin Biotechnol 64:24-31DOI: 10.1016/j.copbio.2019.09.002.

Lin L, Zheng J, Yu Q, Chen W, Xing J, Chen C, and Tian R. 2018. High throughput and accurate serum proteome profiling by integrated sample preparation technology and single-run data independent mass spectrometry analysis. J Proteomics 174:9-16DOI: 10.1016/j.jprot.2017.12.014.

Lin X, Xiong D, Peng YQ, Sheng ZF, Wu XY, Wu XP, Wu F, Yuan LQ, and Liao EY. 2015.Epidemiology and management of osteoporosis in the People's Republic of China: current perspectives. Clin Interv Aging 10:1017-1033DOI: 10.2147/CIA.S54613.

Liu Z, and Lv J. 2019. Integrative Transcriptome and Proteome Analysis Identifies Major Metabolic Pathways Involved in Pepper Fruit Development.J Proteome Res 18:982-994DOI: 10.1021/acs.jproteome.8b00673.

MacLean B, Tomazela DM, Shulman N, Chambers M, Finney GL, Frewen B, Kern R, Tabb DL, Liebler DC, and MacCoss MJ. 2010.Skyline: an open source document editor for creating and analyzing targeted proteomics experiments. Bioinformatics 26:966-968DOI: 10.1093/bioinformatics/btq054.

Martínez-Aguilar MM, Aparicio-Bautista DI, Ramírez-Salazar EG, Reyes-Grajeda JP, De la Cruz-Montoya AH, Antuna-Puente B, Hidalgo-Bravo A, Rivera-Paredez B, Ramírez-Palacios P, Quiterio M, Valdés-Flores M, Salmerón J, and Velázquez-Cruz R. 2019.Serum Proteomic Analysis Reveals Vitamin D-Binding Protein (VDBP) as a Potential Biomarker for Low Bone Mineral Density in Mexican Postmenopausal Women. Nutrients12:pii:nu11122853DOI: 10.3390/nu11122853.

McClung MR, Lewiecki EM, Cohen SB, Bolognese MA, Woodson GC, Moffett AH, Peacock M, Miller PD, Lederman SN, Chesnut CH, Lain D, Kivitz AJ, Holloway DL, Zhang C, Peterson MC, Bekker PJ, and AMG 162 Bone Loss Study Group. 2006. Denosumab in postmenopausal women with low bone mineral density. N Engl J Med 354:821-831.

Meng FH, Li YB, Xiong ZL, Jiang ZM, and Li FM. 2005. Osteoblastic proliferative activity of Epimedium brevicornum Maxim. Phytomedicine 12:189-193.

Miyamoto T, Hirayama A, Sato Y, Koboyashi T, Katsuyama E, Kanagawa H, Fujie A, Morita M, Watanabe R, Tando T, Miyamoto K, Tsuji T, Funayama A, Soga T, Tomita $M$, Nakamura $M$, and Matsumoto $M$. 2018. Metabolomics-based profiles predictive of low bone mass in menopausal women. Bone Rep 9:1118DOI: 10.1016/j.bonr.2018.06.004.

Papadimas GK, Terzis G, Methenitis S, Spengos K, Papadopoulos C, Vassilopoulou S, Kavouras S, Michelakakis H, and Manta P. 2011. Body composition analysis in late-onset Pompe disease. Mol Genet Metab 102:4143DOI: 10.1016/j.ymgme.2010.09.002.

Perez-Riverol Y, Csordas A, Bai J, Bernal-Llinares M, Hewapathirana S, Kundu DJ, Inuganti A, Griss J, Mayer G, Eisenacher M, Pérez E, Uszkoreit J, Pfeuffer J, Sachsenberg T, Yilmaz S, Tiwary S, Cox J, Audain E, Walzer M, Jarnuczak AF, Ternent T, Brazma A, and Vizcaíno JA . 2019. The PRIDE database and related tools and resources in 2019: improving support for quantification data. Nucleic Acids Res 47:D442-D450DOI: 10.1093/nar/gky1106.

Peterson AC, Russell JD, Bailey DJ, Westphall MS, and Coon JJ.2012. Parallel reaction monitoring for high resolution and high mass accuracy quantitative, targeted proteomics. Mol Cell Proteomics 11:14751488DOI: 10.1074/mcp.0112.020131.

Peer] reviewing PDF | (2019:12:44299:1:2:NEW 10 Mar 2020) 
Qaseem A, Forciea MA, McLean RM, and Denberg TD. 2017.Treatment of Low Bone Density or Osteoporosis to Prevent Fractures in Men and Women: A Clinical Practice Guideline Update From the American College of Physicians. Ann Intern Med 166:818-839DOI: 10.7326/M15-1361.

Qian W, Su Y, Zhang Y, Yao N, Gu N, Zhang X, and Yin H. 2018. Secretome analysis of rat osteoblasts during icariin treatment induced osteogenesis. Mol Med Rep 17:6515-6525DOI: 10.3892/mmr.2018.8715.

Shao C, Zhao M, Chen X, Sun H, Yang Y, Xiao X, Guo Z, Liu X, Lv Y, Chen X, Sun W, Wu D, and Gao Y. 2019. Comprehensive Analysis of Individual Variation in the Urinary Proteome Revealed Significant Gender Differences. Mol Cell Proteomics 18:1110-1122DOI: 10.1074/mcp.RA119.001343.

Sheng B, Chu YP, Wong WT, Yau EKC, Chen SPL, and Luk WH. 2017.Improvement of bone mineral density after enzyme replacement therapy in Chinese late-onset Pompe disease patients. BMC Res Notes 10:351DOI: 10.1186/s13104-017-2681-y.

Si L, Winzenberg TM, Jiang Q, Chen M, and Palmer AJ. 2015.Projection of osteoporosis-related fractures and costs in China: 2010-2050. Osteoporos Int26:1929-1937DOI: 10.1007/s00198-015-3093-2.

Thaung Zaw JJ, Howe PRC, and Wong RHX. 2018. Postmenopausal health interventions: Time to move on from the Women's Health Initiative? Ageing Res Rev 48:79-86DOI: 10.1016/j.arr.2018.10.005.

van der Ploeg AT, Clemens PR, Corzo D, Escolar DM, Florence J, Groeneveld GJ, Herson S, Kishnani PS, Laforet P, Lake SL, Lange DJ, Leshner RT, Mayhew JE, Morgan C, Nozaki K, Park DJ, Pestronk A, Rosenbloom B, Skrinar A, van Capelle $\mathrm{Cl}$, van der Beek NA, Wasserstein M, and Zivkovic SA. 2010.A randomized study of alglucosidase alfa in late-onset Pompe's disease. $N$ Engl J Med362:1396-1406DOI: 10.1056/NEJMoa0909859.

Wilmanski T, Rappaport N, Earls JC, Magis AT, Manor O, Lovejoy J, Omenn GS, and Hood L. 2019.Blood metabolome predicts gut microbiome alpha-diversity in humans.Nat Biotechnol 37:1217-1228DOI: 10.1038/s41587-019-0233-9.

You YS, Lin CY, Liang HJ, Lee SH, Tsai KS, Chiou JM, Chen YC, Tsao CK, and Chen JH. 2014.Association between the metabolome and low bone mineral density in Taiwanese women determined by (1)H NMR spectroscopy. $J$ Bone Miner Res 29:212-222DOI: 10.1002/jbmr.2018.

Zhang G, Li J, Zhang J, Liang X, Zhang X, Wang T, and Yin S. 2019. Integrated Analysis of Transcriptomic, miRNA and Proteomic Changes of a Novel Hybrid Yellow Catfish Uncovers Key Roles for miRNAs in Heterosis.Mol Cell Proteomics 18:1437-1453DOI: 10.1074/mcp.RA118.001297.

Zhang L, Liu YZ, Zeng Y, Zhu W, Zhao YC, Zhang JG, Zhu JQ, He H, Shen H, Tian Q, Deng FY, Papasian CJ, and Deng HW. 2016. Network-based proteomic analysis for postmenopausal osteoporosis in Caucasian females. Proteomics 16:12-28DOI: 10.1002/pmic.201500005. 
493

494

495

496

497

498

499

500

501

502

503

504

505

506

507

508

509

510

511

512

513

514

515

516

517

518

519

520

521

522

523

524

525

526

527

528

529

530

531

532

533

\section{Figure and Table Legends}

Figure 1 Experimental workflow. TMT: tandem mass tag, LC-MS/MS: liquid chromatography-tandem mass spectrometry, DDA: data-dependent acquisition, PRM: parallel reaction monitoring.

Figure 2 DXA scan image of the lumbar spine $\left(\mathrm{L}_{1-4}\right)$ and hip for BMD measurement. (A-B) Lumbar and hip in normal BMD group. (C-D) Lumbar and hip in osteopenia group. (E-F) Lumbar and hip in osteoporosis group.

Figure 3 Correlation analysis of age, ageat menopause, duration of menopause, BMI, and BMD.(A) Age was negatively related with BMD values. (B) Age at menopause was positively related with BMD values. (C) Duration of menopause was negatively related with BMD values. (D) BMI was positively related with BMD values.

Figure 4 PCA and PLS-DA analysis of protein expression and DAPs between each of the groups. (A) PCA score plots of proteome data. (B) PLS-DA score plots of proteome data. (C) Number of DAPs between each group. (D) Volcano plot of DAPs between ON versus NC when FC $>1.2$. (E) Volcano plot of DAPs between OP versus NC when FC $>1.2$. (F) Volcano plot of DAPs between OP versus $\mathrm{ON}$ when $\mathrm{FC}>1.2$.

Figure 5 Functional analysis of DAPs between the ON versus NC and OP versus NC groups. (A) Biological process, molecular function, and cellular component annotation of DAPs in the $\mathrm{ON}$ versus NC groups. (B) Biological process, molecular function, and cellular component annotation of DAPs in the OP versus NC groups.

Figure 6 Bi-clustering of the seven DAPs and correlation analysis of DAPs with BMD values. (A) Bi-clustering analysis of seven DAPs in normal BMD and low BMD samples. (B-H) The detailed expression of the seven proteins in each group.P61626: Lysozyme C; A5A0A024R592: Glucosidase; B4DE30: cDNA FLJ51711; Q14554: Protein disulfideisomerase A5; P0C0L4: Complement C4-A; Q658S4: Uncharacterized protein DKFZp666N164; H0Y512: Adipocyte plasma membrane-associated proteinproteins.

Figure 7 Expression patterns of selected DAPs using TMT analysis and PRM validation. (A) Abundance of protein Lysozyme C (P61626). (B) Abundance of protein Glucosidase (A5A0A024R592). (C) Abundance of protein cDNA FLJ51711 (B4DE30). (D) Abundance of protein Protein disulfideisomerase (Q14554). ${ }^{*} P<0.05$ between two groups; ${ }^{*} P<0.01$ between two groups.

Supplementary Figure 1 Correlation analysis of biochemical detection and BMD values.

Peer) reviewing PDF | (2019:12:44299:1:2:NEW 10 Mar 2020) 
534 Supplementary Figure 2 The MS/MS spectra for the seven DAPs.

535

536

537

538

539

540

541

542

543

544
Supplementary Figure 3 Correlation analysis of DAPs with BMD values. (A) The abundance of protein Lysozyme C (P61626) was positively related with BMD values. (B-D) The abundance of proteins Glucosidase (A5A0A024R592), cDNA FLJ51711 (B4DE30), and Protein disulfideisomerase (Q14554) was negatively related to BMD values. (E-G) The abundance of proteins Complement C4-A (P0C0L4), Uncharacterized protein DKFZp666N164 (Q658S4), and Adipocyte plasma membrane-associated proteinproteins (H0Y512) was not significantly related to $\mathrm{BMD}$ values. 
545 Table 1 Participant characteristics.

\begin{tabular}{|c|c|c|c|c|c|c|c|c|c|c|}
\hline Group & $\mathbf{n}$ & Age & M-age & BMI & $\begin{array}{l}\text { Vitamin } \\
(\mathrm{ng} / \mathrm{mL})\end{array}$ & $\begin{array}{l}\text { ALP } \\
(\mathrm{U} / \mathrm{L})\end{array}$ & $\begin{array}{c}\text { P1NP } \\
(\mathrm{ng} / \mathrm{mL})\end{array}$ & $\begin{array}{c}\text { СТX } \\
(\mathrm{ng} / \mathrm{mL})\end{array}$ & $\begin{array}{c}\text { BMD } \\
\left(\mathrm{g} / \mathrm{cm}^{2}\right)\end{array}$ & T score \\
\hline Normal control & 18 & $55.22 \pm 5.31$ & $48.06 \pm 2.70$ & $25.02 \pm 2.86$ & $17.81 \pm 4.36$ & $99.33 \pm 28.85$ & $77.90 \pm 26.55$ & $0.44 \pm 0.15$ & $0.92 \pm 0.11$ & $-0.47 \pm 0.57$ \\
\hline Osteopenia & 18 & $56.72 \pm 4.92$ & $47.11 \pm 1.71$ & $24.71 \pm 2.58^{\#}$ & $19.05 \pm 5.54$ & $108.39 \pm 37.47$ & $70.98 \pm 26.49$ & $0.41 \pm 0.14$ & $0.81 \pm 0.06^{\#}$ & $-1.94 \pm 0.39^{\#}$ \\
\hline Osteoporosis & 18 & $58.33 \pm 5.40$ & $46.83 \pm 2.07$ & $21.89 \pm 1.64^{\# \triangle}$ & $19.17 \pm 7.27$ & $109.61 \pm 27.86$ & $90.93 \pm 42.68$ & $0.49 \pm 0.18$ & $0.61 \pm 0.24^{\# \triangle}$ & $-4.50 \pm 0.26^{\# \triangle}$ \\
\hline Total & 54 & $56.76 \pm 5.27$ & $47.32 \pm 2.21$ & $23.87 \pm 2.76$ & $18.69 \pm 5.77$ & $105.78 \pm 31.43$ & $79.94 \pm 33.25$ & $0.45 \pm 0.16$ & $0.78 \pm 0.20$ & $-2.31 \pm 1.73$ \\
\hline
\end{tabular}

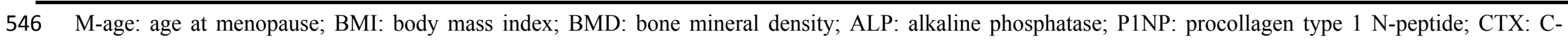

547 terminal cross-linking telopeptide of type 1 collagen. ${ }^{\#}$ Compared with the NC group, $P<0.01 ;{ }^{\Delta}$ compared with the ON group, $P<0.01$.

548

549

550 Table 2 Changes in the plasma proteins between low BMD and normal controls. 


\section{Figure 1}

\section{Experimental workflow.}

TMT: tandem mass tag, LC-MS/MS: liquid chromatography-tandem mass spectrometry, DDA: data-dependent acquisition, PRM: parallel reaction monitoring. 

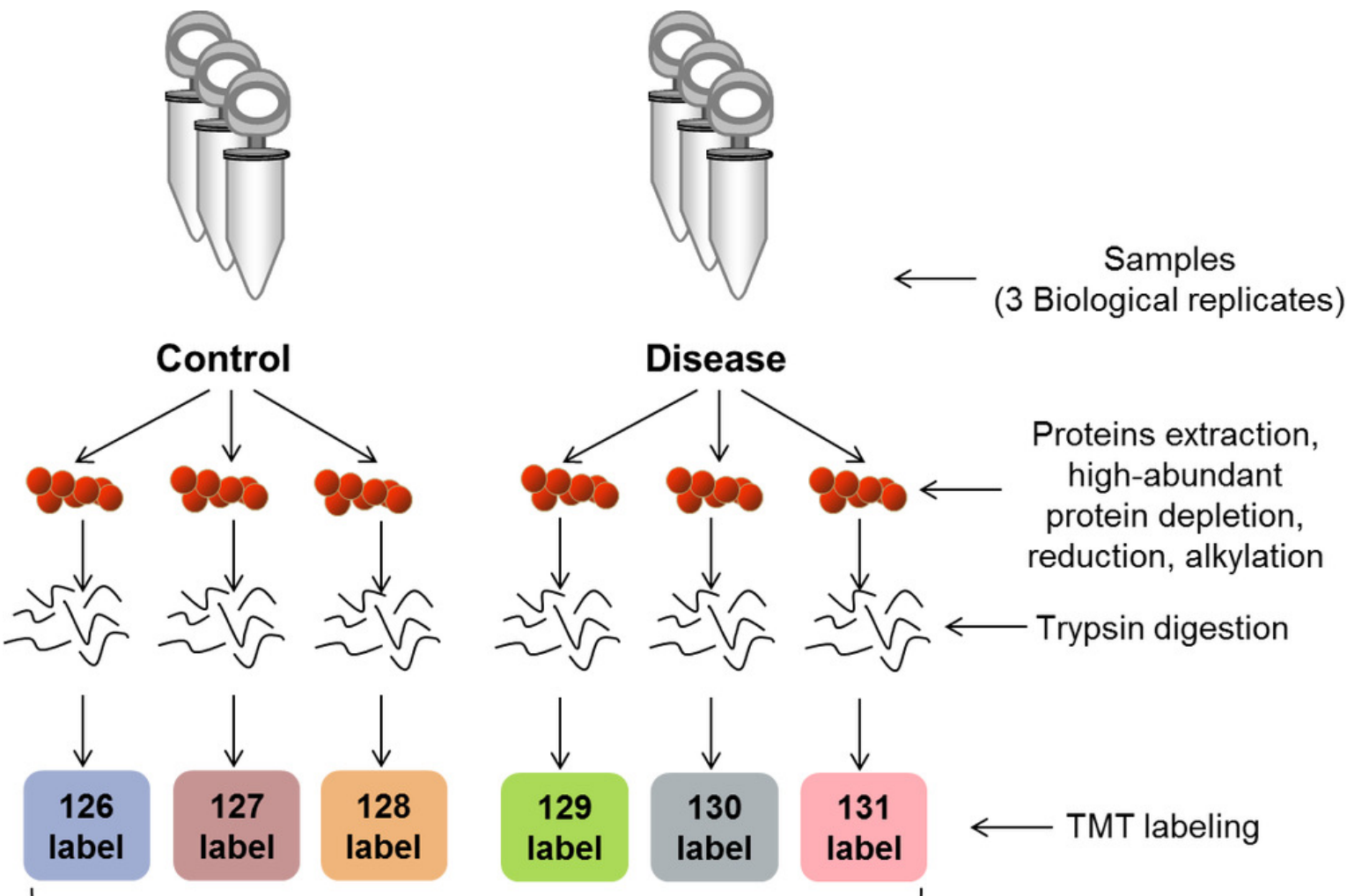

Proteins extraction, high-abundant protein depletion,

$\downarrow \quad$ reduction, alkylation $\widetilde{v} \widehat{\sim} \widetilde{r} \leftarrow$ Trypsin digestion

\section{Combined labeled peptides}

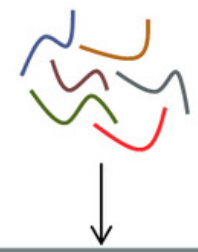

High $\mathrm{pH}$ reversed-phase peptide fractionation

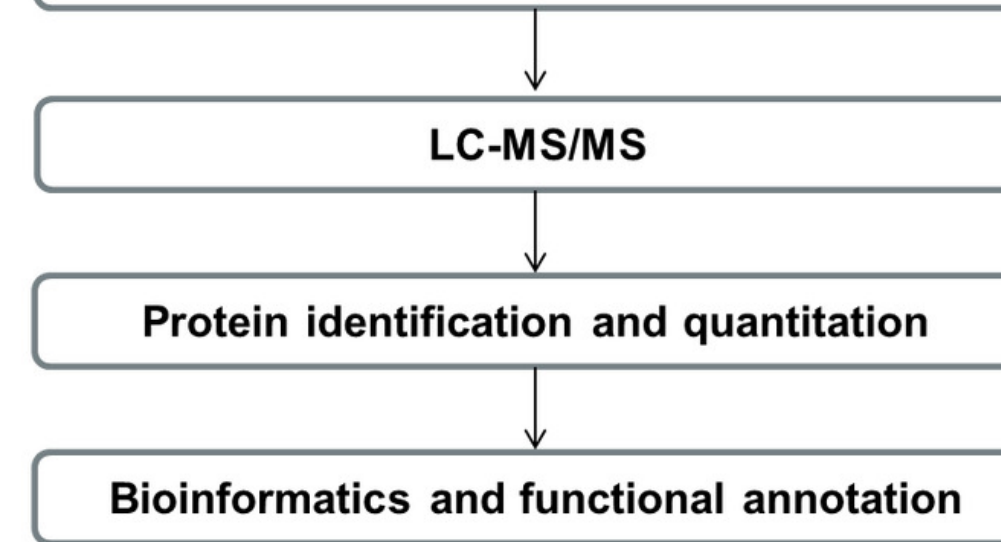


Figure 2

DXA scan image of the lumbar spine (L1-4) and hip for BMD measurement.

(A-B) Lumbar and hip in normal BMD group. (C-D) Lumbar and hip in osteopenia group. (E-F) Lumbar and hip in osteoporosis group. 

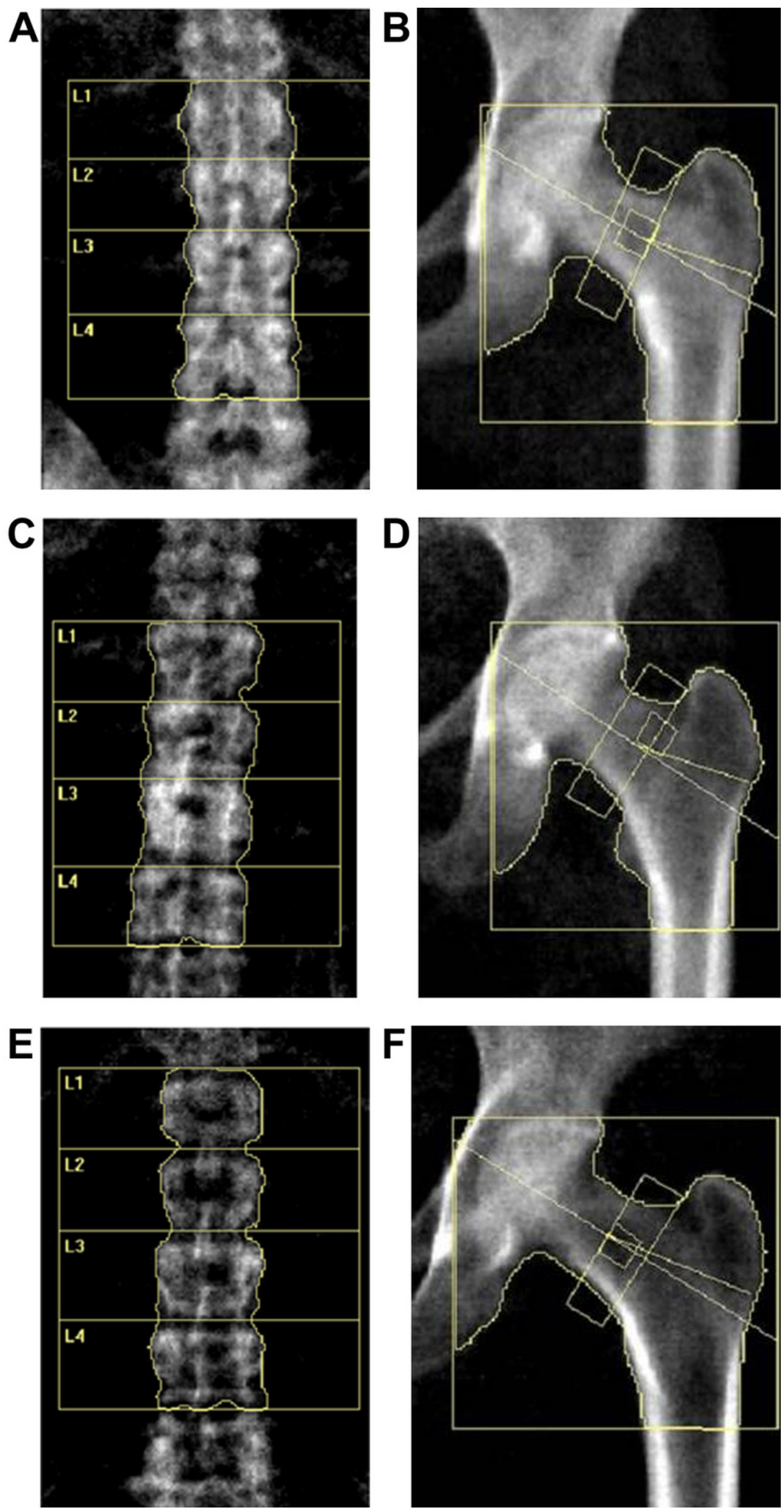

Peer) reviewing PDF | (2019:12:44299:1:2:NEW 10 Mar 2020) 
Figure 3

Correlation analysis of age, age at menopause, duration of menopause, BMI, and BMD.

(A) Age was negatively related with BMD values. (B) Age at menopause was positively related with BMD values. (C) Duration of menopause was negatively related with BMD values. (D) BMI was positively related with BMD values.
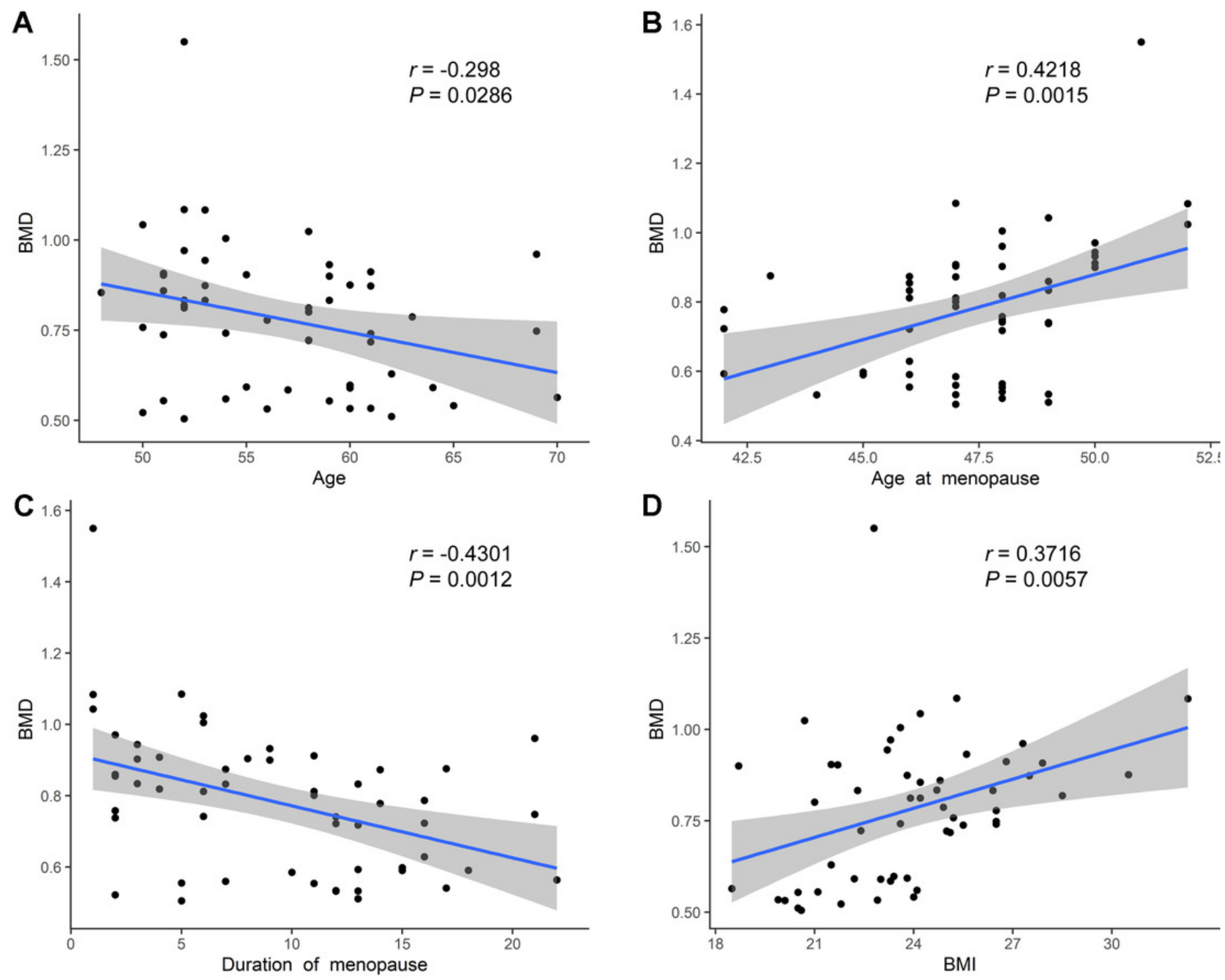
Figure 4

PCA and PLS-DA analysis of protein expression and DAPs between each of the groups.

(A) PCA score plots of proteome data. (B) PLS-DA score plots of proteome data. (C) Number of DAPs between each group. (D) Volcano plot of DAPs between ON versus NC when FC $>1.2$.

(E) Volcano plot of DAPs between OP versus NC when FC $>1.2$. (F) Volcano plot of DAPs between $\mathrm{OP}$ versus $\mathrm{ON}$ when $\mathrm{FC}>1.2$.
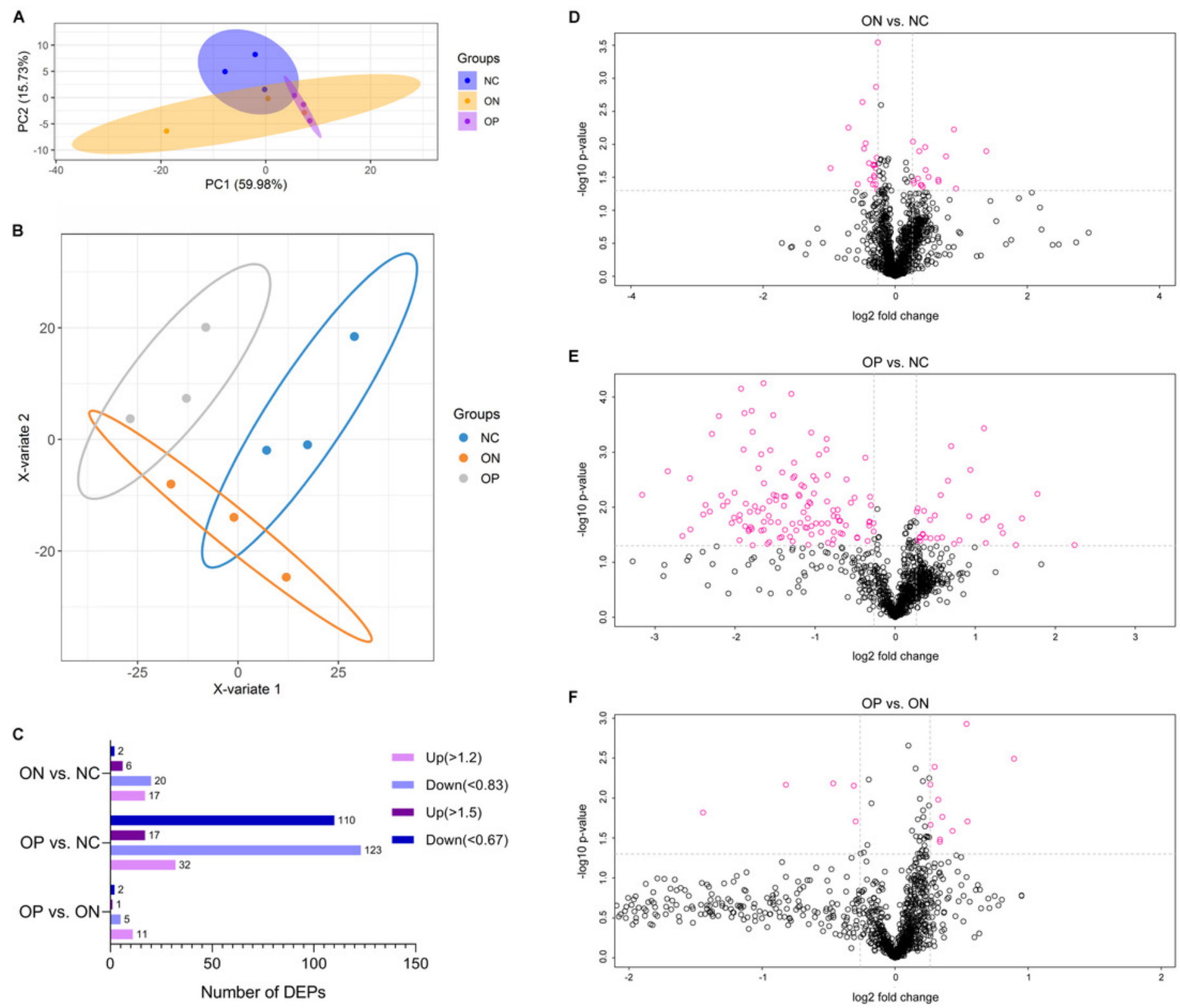


\section{Figure 5}

Functional analysis of DAPs between the ON versus NC and OP versus NC groups.

(A) Biological process, molecular function, and cellular component annotation of DAPs in the ON versus NC groups. (B) Biological process, molecular function, and cellular component annotation of DAPs in the OP versus NC groups. 
A

ON vs. NC

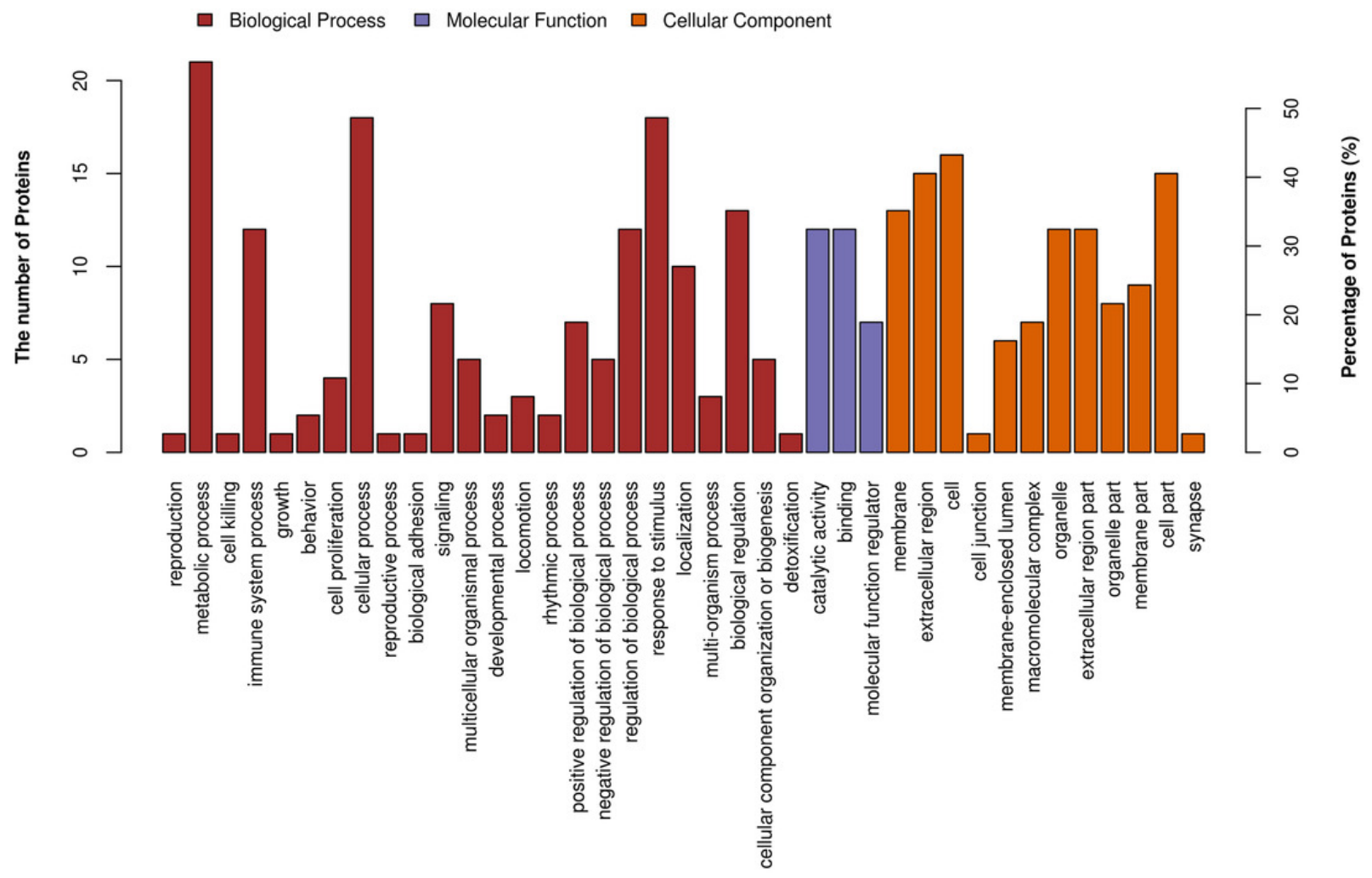

B

OP vs. NC

- Biological Process $\square$ Molecular Function $\square$ Cellular Component

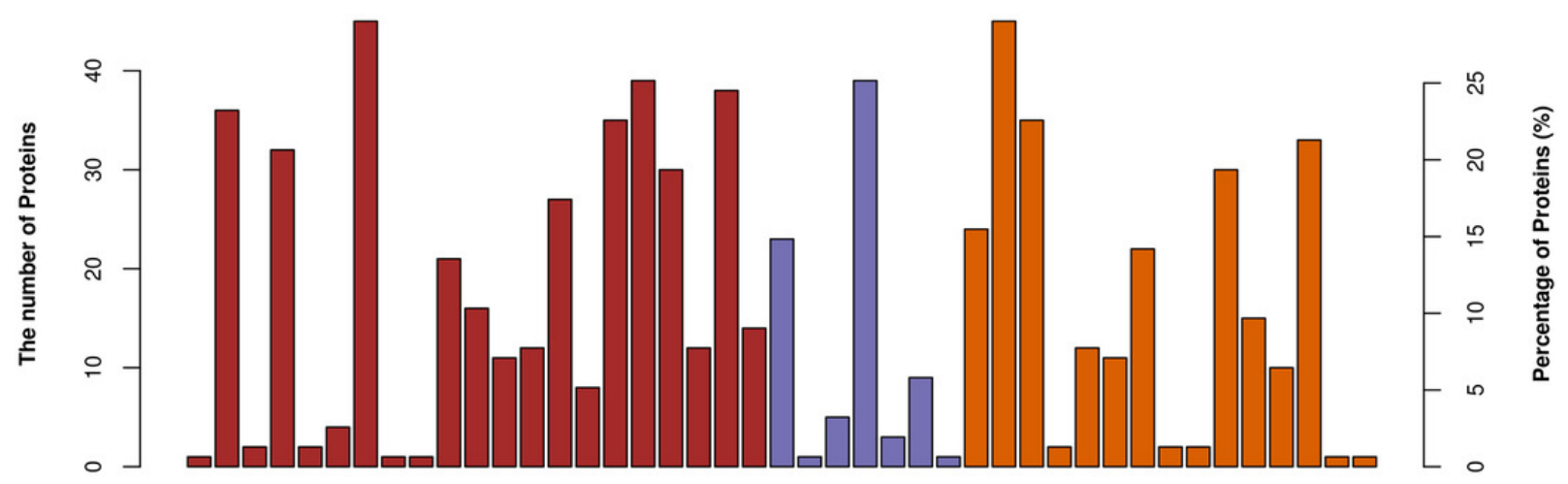

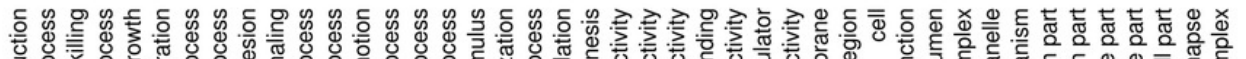

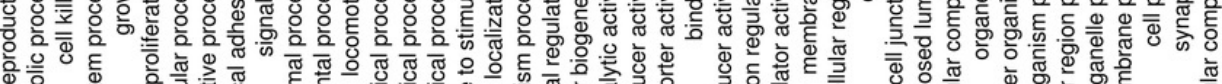

$\begin{array}{lllll} & \\ 0\end{array}$ 


\section{Figure 6}

Bi-clustering of the seven DAPs and correlation analysis of DAPs with BMD values.

(A) Bi-clustering analysis of seven DAPs in normal BMD and low BMD samples. (B-H) The detailed expression of the seven proteins in each group.P61626: Lysozyme C; A5A0A024R592: Glucosidase; B4DE30: cDNA FLJ51711; Q14554: Protein disulfideisomerase A5; POC0L4: Complement C4-A; Q658S4: Uncharacterized protein DKFZp666N164; H0Y512: Adipocyte plasma membrane-associated proteinproteins. 


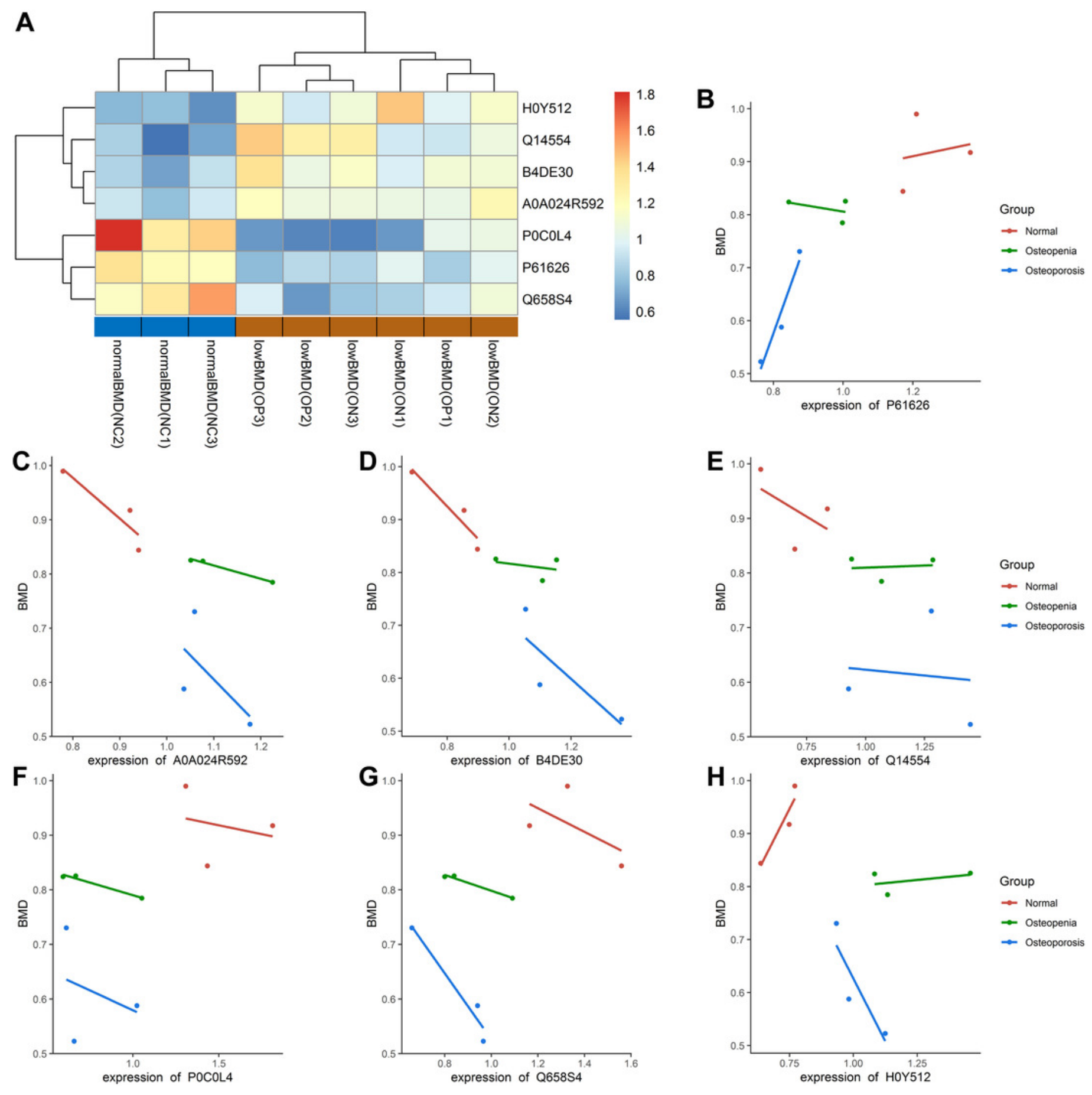


Figure 7

Expression patterns of selected DAPs using TMT analysis and PRM validation.

(A) Abundance of protein Lysozyme C (P61626). (B) Abundance of protein Glucosidase (A5A0A024R592). (C) Abundance of protein CDNA FLJ51711 (B4DE30). (D) Abundance of protein Protein disulfideisomerase (Q14554). ${ }^{*} P<0.05$ between two groups; $* * P<0.01$ between two groups.

A

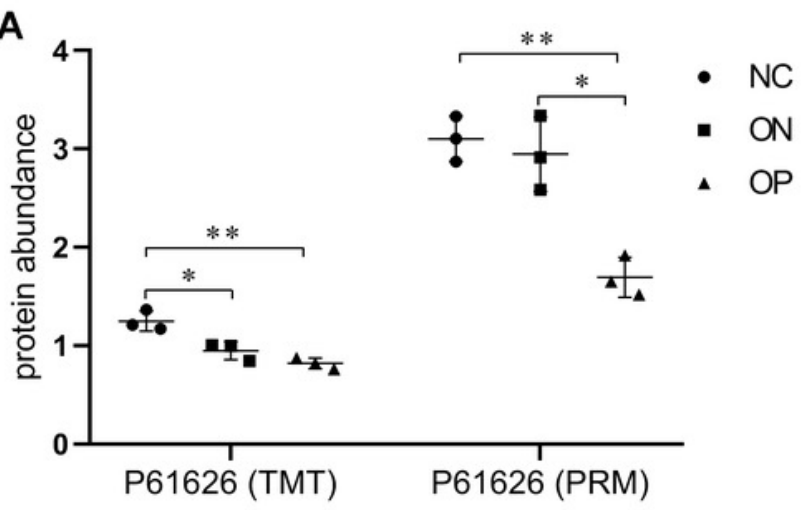

C

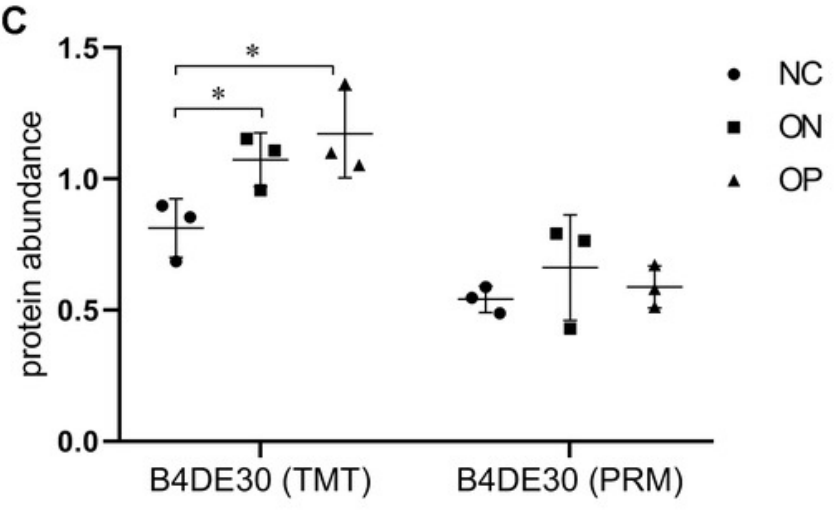

B
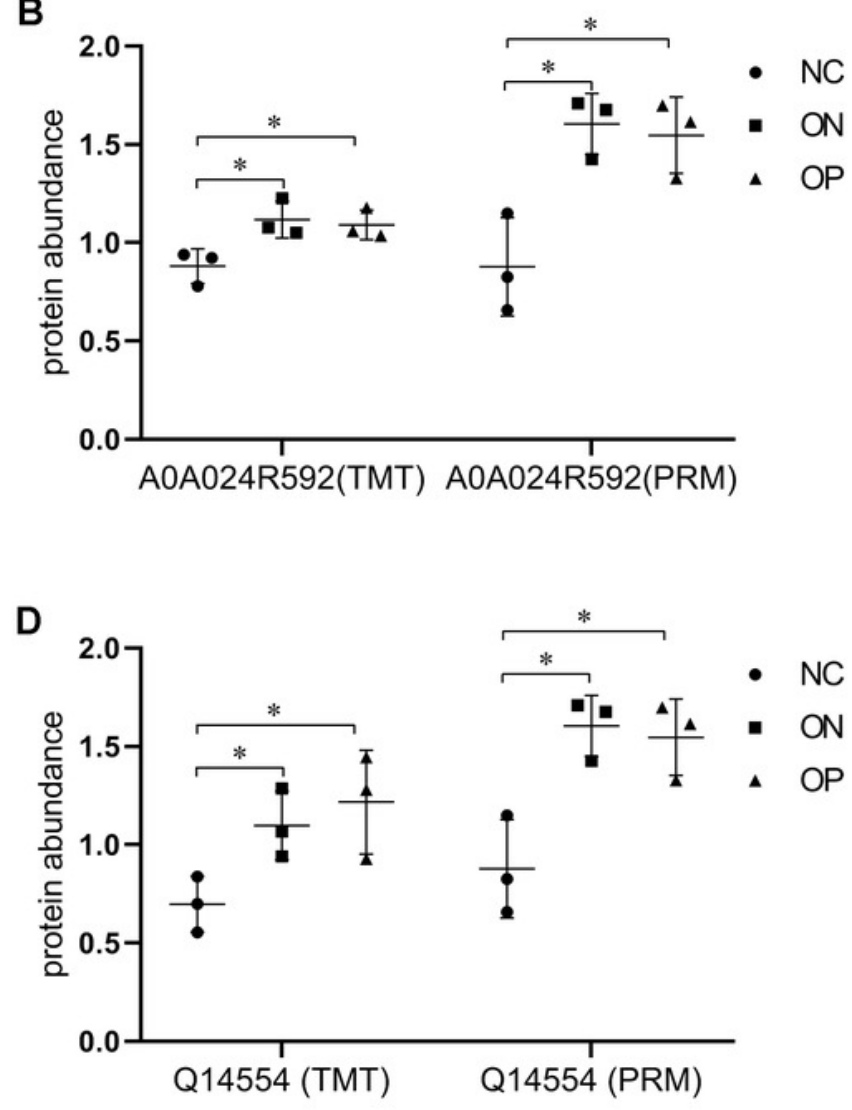
Table $\mathbf{1}$ (on next page)

Participant characteristics 
1 Table 1 Participant characteristics.

\begin{tabular}{|c|c|c|c|c|c|c|c|c|c|c|}
\hline Group & $\mathbf{n}$ & Age & M-age & BMI & $\begin{array}{l}\text { Vitamin } \\
(\mathrm{ng} / \mathrm{mL})\end{array}$ & $\begin{array}{l}\text { ALP } \\
(\mathrm{U} / \mathrm{L})\end{array}$ & $\begin{array}{c}\text { P1NP } \\
\text { (ng/mL) }\end{array}$ & $\begin{array}{c}\text { CTX } \\
(\mathrm{ng} / \mathrm{mL})\end{array}$ & $\begin{array}{c}\text { BMD } \\
\left(\mathrm{g} / \mathrm{cm}^{2}\right)\end{array}$ & T score \\
\hline Normal control & 18 & $55.22 \pm 5.31$ & $48.06 \pm 2.70$ & $25.02 \pm 2.86$ & $17.81 \pm 4.36$ & $99.33 \pm 28.85$ & $77.90 \pm 26.55$ & $0.44 \pm 0.15$ & $0.92 \pm 0.11$ & $-0.47 \pm 0.57$ \\
\hline Osteopenia & 18 & $56.72 \pm 4.92$ & $47.11 \pm 1.71$ & $24.71 \pm 2.58^{\#}$ & $19.05 \pm 5.54$ & $108.39 \pm 37.47$ & $70.98 \pm 26.49$ & $0.41 \pm 0.14$ & $0.81 \pm 0.06^{\#}$ & $-1.94 \pm 0.39^{\#}$ \\
\hline Osteoporosis & 18 & $58.33 \pm 5.40$ & $46.83 \pm 2.07$ & $21.89 \pm 1.64^{\# \triangle}$ & $19.17 \pm 7.27$ & $109.61 \pm 27.86$ & $90.93 \pm 42.68$ & $0.49 \pm 0.18$ & $0.61 \pm 0.24^{\# \triangle}$ & $-4.50 \pm 0.26^{\# \triangle}$ \\
\hline Total & 54 & $56.76 \pm 5.27$ & $47.32 \pm 2.21$ & $23.87 \pm 2.76$ & $18.69 \pm 5.77$ & $105.78 \pm 31.43$ & $79.94 \pm 33.25$ & $0.45 \pm 0.16$ & $0.78 \pm 0.20$ & $-2.31 \pm 1.73$ \\
\hline
\end{tabular}

2 M-age: age at menopause; BMI: body mass index; BMD: bone mineral density; Vitamin: 25-hydroxyvitamin D; ALP:alkaline phosphatase; P1NP: procollagen

3 type $1 \mathrm{~N}$-peptide; ctx: C-terminal cross-linking telopeptide of type 1 collagen. ${ }^{*}$ compared with the NC group, $P<0.01 ;{ }^{\Delta}$ compared with the ON group, $P<0.01$. 
Table 2 (on next page)

Changes in the plasma proteins between low BMD and normal controls. 
1 Table 2 Changes in the plasma proteins between low BMD and normal controls.

\begin{tabular}{llrrrrrrr}
\hline \multirow{2}{*}{ Accession } & \multicolumn{2}{c}{ Protein name (description) } & Average & Average & Average & \multicolumn{2}{c}{ ON vs NC } & \multicolumn{2}{c}{ OP vs NC } \\
\cline { 6 - 9 } & & NC & ON & OP & FC & $P$ & FC & $P$ \\
\hline P61626 & Lysozyme C & 1.249 & 0.949 & 0.820 & 0.760 & 0.019 & 0.657 & 0.003 \\
A0A024R592 & Glucosidase & 0.880 & 1.118 & 1.091 & 1.270 & 0.033 & 1.239 & 0.035 \\
B4DE30 & cDNA FLJ51711 & 0.813 & 1.073 & 1.172 & 1.320 & 0.041 & 1.442 & 0.037 \\
Q14554 & Protein disulfide isomerase A5 & 0.697 & 1.098 & 1.217 & 1.575 & 0.037 & 1.746 & 0.040 \\
P0C0L4 & Complement C4-A & 1.516 & 0.771 & 0.764 & 0.508 & 0.023 & 0.504 & 0.020 \\
Q658S4 & Uncharacterized protein DKFZp666N164 & 1.350 & 0.910 & 0.855 & 0.674 & 0.040 & 0.633 & 0.031 \\
H0Y512 & Adipocyte plasma membrane-associated protein & 0.719 & 1.225 & 1.013 & 1.704 & 0.015 & 1.410 & 0.014 \\
\hline
\end{tabular}

\section{2}

3

4 